\title{
Carbon dioxide emissions rebound from the COVID-19 pandemic
}

\author{
Steven J. Davis ${ }^{1,2}$, Zhu Liu ${ }^{3}$, Zhu Deng 3 , Biqing Zhu ${ }^{3}$, Piyu Ke ${ }^{3}$, Taochun Sun $^{3}$, Rui Guo ${ }^{3}$, \\ Chaopeng Hong ${ }^{4}$, Bo Zheng ${ }^{4}$, Yilong Wang 5 , Olivier Boucher ${ }^{6}$, Pierre Gentine $^{7}$, and \\ Philippe Ciais $^{8}$
}

${ }^{1}$ Department of Earth System Science, University of California at Irvine, Irvine, CA, USA

${ }^{2}$ Department of Civil and Environmental Engineering, University of California at Irvine, Irvine, CA, USA

${ }^{3}$ Department of Earth System Science, Tsinghua University, Beijing, China

${ }^{4}$ Department of Earth System Science, Tsinghua University, Shenzhen, China

${ }^{5}$ Key Laboratory of Land Surface Pattern and Simulation, Institute of Geographical Sciences and Natural Resources Research, Chinese Academy of Sciences, Beijing, China

${ }^{6}$ Institut Pierre-Simon Laplace, Sorbonne Université / CNRS, Paris, France

${ }^{7}$ Department of Earth and Environmental Engineering, Columbia University, New York, NY, USA

${ }^{8}$ Laboratoire des Sciences du Climat et de l'Environnement, LSCE/IPSL, CEA-CNRS- UVSQ, Université ParisSaclay, Gif-sur-Yvette, France

*Corresponding authors' email addresses: sjdavis@uci.edu, zhuliu@tsinghua.edu.cn, philippe.ciais@cea.fr

\section{Main Text:}

1066 words (excluding references, and figure legends)

Figures $1-2$ 


\section{Global $\mathrm{CO}_{2}$ emissions have rebounded strongly from the historic decrease caused by the COVID-19 pandemic in 2020 . We project 2021 emissions will be only $0.9 \%$ less than the record levels of 2019. In particular, power- and industry-related emissions in China and India have increased relative to 2019, and the carbon intensity of electricity used worldwide has returned to its pre-pandemic level. Is this rebound a sign of fossil energy resuming growth or a final fleeting surge at the start of a long decline?}

Four years after the Paris Agreement was adopted, global fossil fuel emissions reached a record high of more than $35 \mathrm{Gt} \mathrm{CO}_{2}$ in 2019 ${ }^{1}$. Then widespread disruptions in human activities and energy use caused by the COVID-19 pandemic led to an unprecedented $6 \%$ drop in 2020 emissions, to $33.3 \mathrm{Gt} \mathrm{CO}_{2}^{2,3}$. In turn, this remarkable decrease in emissions set analysts speculating about longer-term changes in the ways energy is generated and used worldwide, and - given persistently high growth rates of renewable energy and increasingly ambitious climate commitments - about whether 2019 could even have been the high-water mark of global emissions $^{4-6}$. Specifically, analysts expect the long-term decline of fossil energy to begin when annual increases in renewables and other non-fossil sources of energy entirely meet new energy demand - a time that may be hastened by both pandemic-related decreases in energy demand ($4 \%$ in global energy demand in $2020^{7}$ ) and stimulus-driven increases in the growth rate of renewable energy.

However, the latest estimates of the Carbon Monitor (an international collaboration begun in 2020 to track global, regional, and sectoral emissions in near real-time), based on assimilated activity data from major countries and sectors ${ }^{8}$ reveal a strong rebound of emissions in 2021: emissions between January 1 and September 30 of 2021 are only $0.9 \%$ less than those of the same period in 2019 (Fig. 1). If this difference is maintained over the next few months, total global emissions in 2021 will reach $35.0 \pm 0.2 \mathrm{Gt} \mathrm{CO}_{2}-\mathrm{a} 5.1 \%$ increase on the year that will bring emissions just shy of 2019 levels. Our estimate is very similar to recent, independent IEA projections of a $4.8 \%$ increase in $2021^{7}$. These emissions are an integrated measure of the race between rebound and non-fossil sources of energy, and at a global level they suggest that energy demand is resurgent and outpacing efforts to "build back better."

Yet Figure 1c shows the patterns of emissions decrease and rebound 2019-2020 have varied widely across regions and sectors. The emissions decrease in the U.S. was larger than in any 
other individual country $(-9.4 \%$ in 2020$)$, but the rebound has also been strong (projected to be $+7.8 \%$ in 2021; blue bars). The combined decreases in 2020 emissions from smaller countries were also quite large (brown bars), but in contrast there has been very little recovery in these countries' emissions in 2021. Meanwhile, in India, Russia and Brazil, 2021 emissions are on track to exceed those in 2019 by $1.7 \%, 2.9 \%$ and $9.4 \%$, respectively (green, orange and yellow bars), and in China annual emissions did not decrease at all, but grew by $0.9 \%$ in 2020 and are projected to increase by a further $6.4 \%$ in 2021 (red bars). These differences are noteworthy indicators of both the magnitude and persistence of pandemic-related disruptions in different regions. In particular, 2021 emissions would have very likely exceeded 2019 emissions but for the mostly low-income "Rest of World" countries that have not recovered from the pandemic.

Similarly, Figure 1d shows that emissions from road transportation and aviation decreased substantially in 2020 (road transportation by $-10.9 \%$ in orange, and - domestic and international aviation by $-30.8 \%$ and $-56.0 \%$ in dark green and blue bars, respectively), and are on track to remain below 2019 levels in 2021 (though they have all rebounded somewhat). Meanwhile, power and industry sector emissions are projected to fully recover from substantial drops during 2020, with their emissions increasing by $2.1 \%$ and $0.7 \%$ relative to 2019 , respectively (light green and yellow bars). Increases in power-related emissions relative to 2019 underscores the strong rebound of fossil energy within the sector most amenable to decarbonization.

Further analyzing the changes in power-related emissions, we see that the largest decreases in the second quarter of 2020 were driven by decreases in the carbon intensity of electricity (i.e. decreases in the relative shares of fossil fuel generation; Figs. $2 \mathrm{a}$ and 2b), but that corresponding increases in carbon intensity also underlie the increases in the power sector emissions in the fourth quarter of 2020 and the second quarter of 2021 (Fig. 2a). This suggests that the carbon intensity of the global power sector is highly sensitive to electricity demand at present — when demand declined, use of fossil fuels plummeted, but as demand has rebounded, fossil generators have just as quickly resumed operations.

Thus, the latest estimates cast doubt upon the prospect that 2019 was the high point of global fossil fuel emissions. Although solar and wind energy continue to grow much faster than fossil sources (e.g., renewables accounted for $83 \%$ of new power capacity in $2020^{9}$ and only China installed substantial new coal capacity ${ }^{10}$ ), overall energy use and emissions are recovering rapidly in many places and some tallies show that more energy-related stimulus has gone to 
fossil fuels than to renewables ${ }^{9}$. Despite this, decades-long trends in non-fossil energy and large fluctuations in the carbon intensity of electricity during the pandemic indicate that the long dominance of fossil fuels is now precarious. When and how quickly the use of such fuels will decline, however, will depend upon how successful countries are in moderating increases in energy demand, curtailing further growth of fossil energy ${ }^{11}$, and accelerating growth of nonfossil energy sources. Although many of the mitigation scenarios recently produced by integrated assessment models, energy system models, and industry groups project fossil emissions to increase for years to come ${ }^{12,13}$, such models have routinely and systematically underestimated the falling costs and related growth of modern renewables such as solar photovoltaics ${ }^{14,15}$ — and may be doing so again ${ }^{16}$. If so, as world leaders gather in Glasgow for COP26 in a year with near record high global $\mathrm{CO}_{2}$ emissions, their most important task may be to remove barriers to the deployment of renewables. In any event, the coming year should reveal whether 2021 was a fleeting spike at the beginning of a long downward trend in emissions or whether fossil fuel emissions have more years of growing to do. [1066 words] 


\section{References}

1 Friedlingstein, P. et al. Global Carbon Budget 2020. Earth System Science Data 12, 3269-3340 (2020).

2 Quéré, C. L. et al. Temporary reduction in daily global CO2 emissions during the COVID-19 forced confinement. Nature Climate Change 10, 647-653 (2020).

3 Liu, Z. et al. Near-real-time monitoring of global CO2 emissions reveals the effects of the COVID-19 pandemic. Nature Communications 11, 5172 (2020).

$4 \quad$ Kikstra, J. S. et al. Climate mitigation scenarios with persistent COVID-19-related energy demand changes. Nature Energy, doi:10.1038/s41560-021-00904-8 (2021).

5 Quéré, C. L. et al. Fossil CO2 emissions in the post-COVID-19 era. Nature Climate Change 11, 197-199 (2021).

6 Henbest, S., Kimmel, M., Callens, J. \& Vasdev, A. New Energy Outlook. (BloombergNEF, 2021).

$7 \quad$ IEA. Global Energy Review 2021. (International Energy Agency, Paris, France, 2021).

8 Liu, Z. et al. Carbon Monitor, a near-real-time daily dataset of global CO2 emission from fossil fuel and cement production. Scientific Data 7, 392 (2020).

9 Renewables 2021 Global Status Report. (REN21, 2021).

10 Boom and bust: Tracking the global coal plant pipeline. (Global Energy Monitor, Sierra Club, CREA, Climate RIsk Horizons, GreenID, Ekosfer, 2021).

11 Littlecott, C. et al. No New Coal by 2021: The collapse of the global coal pipeline. 60 (E3G, 2021).

12 IEA. World Energy Outlook. (International Energy Agency, 2021).

13 DeAngelo, J. et al. Energy systems in scenarios at net-zero CO2 emissions. Nature Communications 12, 6096 (2021).

14 Davis, S. J. Predicting unpredictability. Nature Energy 3, 257-258 (2018).

15 Creutzig, F. et al. The underestimated potential of solar energy to mitigate climate change. Nature Energy 2, 17140 (2017).

16 Way, R., Ives, M., Mealy, P. \& Farmer, J. D. Empirically grounded technology forecasts and the energy transition. (Oxford Martin School, Oxford, UK, 2021). 

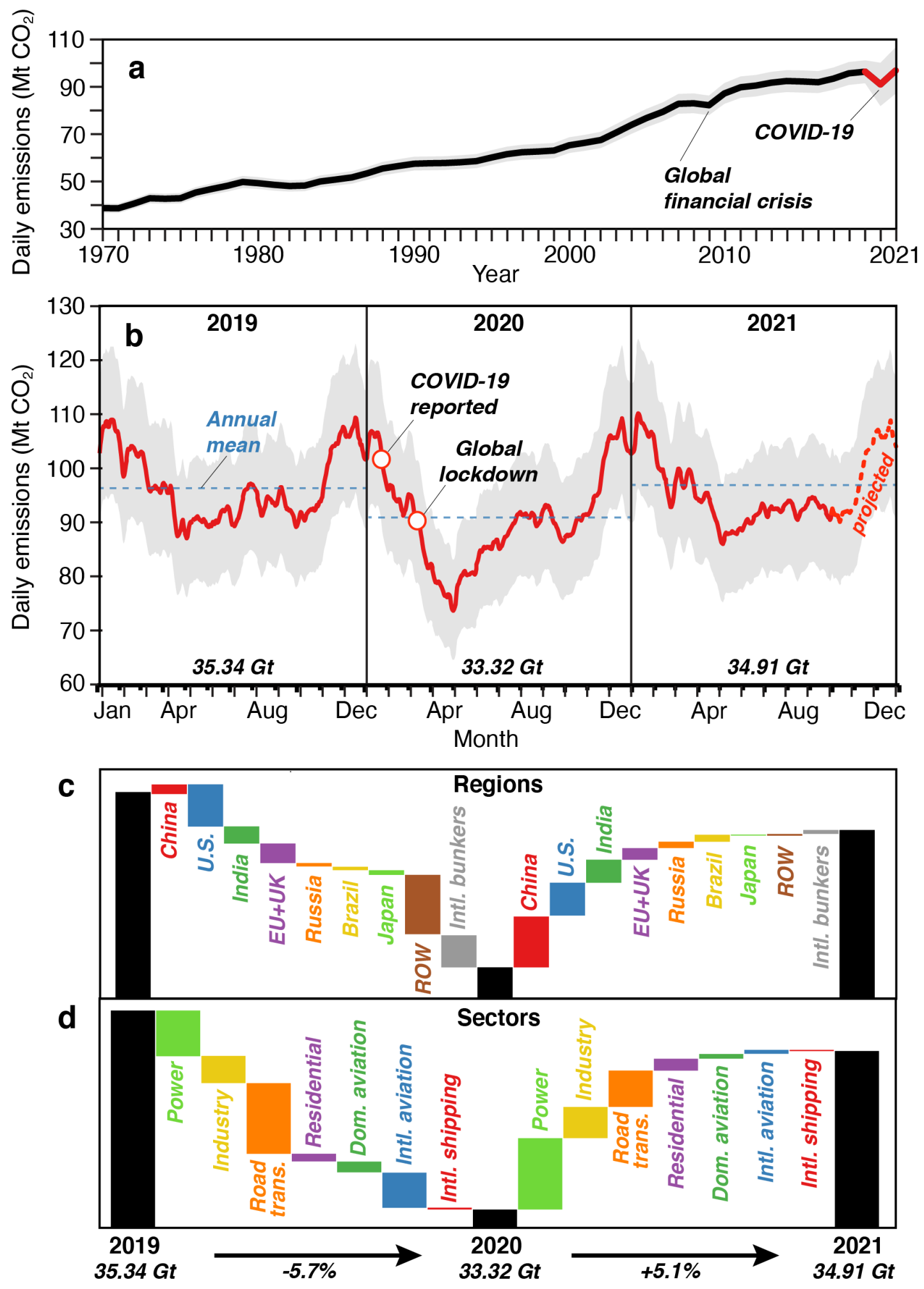

Figure 1 | Trends in global $\mathrm{CO}_{2}$ emissions. Long-term increases in global $\mathrm{CO}_{2}$ emissions have been punctuated by decreases in some years related to global crises such as the COVID-19 pandemic (a). The pandemic-related decreases in emissions were greatest in mid-2020 and have rebounded strongly in 2021 (b), though changes in emissions have varied across regions and sectors $(\mathbf{c}, \mathbf{d})$. 

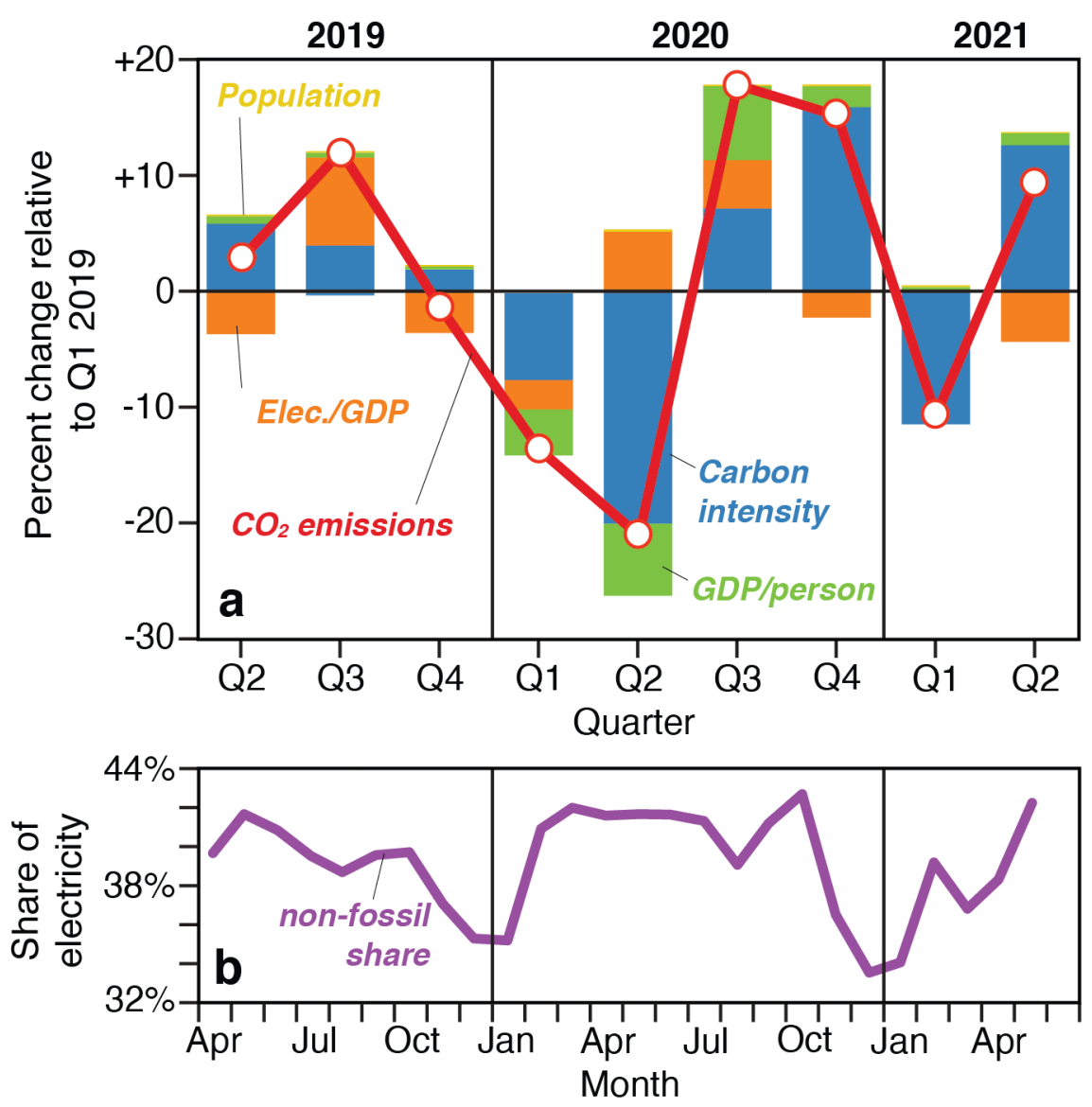

Figure 2 Decomposition of changes in power sector emissions. Changes in electricity-related $\mathrm{CO}_{2}$ emissions (a, red line) relative to the beginning of 2019 have most often been driven by changes in carbon intensity $\left(\mathrm{CO}_{2}\right.$ per unit electricity consumed; blue bars), though the largest decrease during the second quarter of 2020 and rebound in the subsequent third quarter were also influenced by changes in economic activity (GDP per person; green bars). In turn, the changes in quarterly carbon intensity correspond to variations in the share of electricity from non-fossil sources (i.e. renewables and nuclear; b). 